\title{
The Great Imposter: A case Report of IgG4 RD -Hypertrophic Pachymeningitis with Skull Lytic lesion and Pulmonary Nodules
}

\author{
Muhammad Wadiwala ${ }^{1}$, Liaquat $\mathrm{Ali}^{1}$, Adnan Khan ${ }^{2}$, and Mohammed alhatou ${ }^{1}$ \\ ${ }^{1}$ Hamad Medical Corporation \\ ${ }^{2}$ Weill Cornell Medical College in Qatar
}

November 10, 2021

\begin{abstract}
Immunoglobulin G4-related disease is an inflammatory condition with unique clinical, serological, and pathological features. Here, we report a diagnostic challenging clinical case. Histopathology was used to diagnose his Ig4RD. This unique inature reinforces the fact that it is crucial to consider the diagnosis of IgG4-RD in those presenting with pachymeningiti
\end{abstract}

\section{Introduction}

IgG4 related disease immunoglobulin G4 (IgG4)-related disease is recently recognized as a multi-systemic syndrome characterized by an immune-mediated fibroinflammatory condition with unique clinical, serological, and pathological features (1). It can involve any organ system and has the tendency to form multiple stupefactive lesions (1). It was first reported in the pancreas in early 2000 but gradually has been reported in other sites $(2,3)$. Pancreatic salivary glands, bile duct, and lacrimal glands are typically affected. The diagnosis of IgG4-RD requires the identification of characteristic findings upon biopsy of affected tissue which is interpreted in the context of clinical, serologic, and radiologic data. Ig4RD is a steroid-responsive disease and early recognition, and treatment is important because of the indolent nature of the condition and the risk of progression from an inflammatory stage to poorly responsive fibrotic disease and serious organ damage. Most patients experience disease flares during or after glucocorticoid tapers (4). Thus, additional immunosuppressive therapy may also be required.

Neurological presentation is less common and usually presents as hypophysitis followed by hypertrophic pachymeningitis (HP) (3). Hypertrophic pachymeningitis is a rare inflammatory disorder that causes localized or diffuse thickening of intracranial dura matter and has a broad range of neoplastic, chronic inflammatory, infectious, and hemorrhagic conditions associated with it and can therefore be a diagnostic challenge $(1,3)$. When no cause is found it is termed as idiopathic hypertrophic pachymeningitis. A dural biopsy is usually essential for a definitive diagnosis. Clinical manifestations of the disease are subject to localization or complications due to the compression and/or infiltration of neurologic and/or vascular adjacent structures. It has been suggested that IgG4-related sclerosing disease represents a subset of cases previously diagnosed as idiopathic hypertrophic pachymeningitis (5). Headache, cranial nerve palsy, and ataxia are the most common presentations (6). Seizures as chief presentations are uncommonly reported in the literature $(5,6)$.

Here we report highlights a unique, intricate case of a young male presented to our hospital with presentations imitating a variety of pathological conditions After undergoing a diagnostic workup, he was eventually diagnosed to have histologically proven Ig $4 \mathrm{RD}$ systemic disease with meningeal involvement, which is a rare entity.

\section{Case report}


We describe a case of a 25-year-old South Asian gentleman with no past medical history who suffered a seizure during an air flight and brought to the hospital. The event started with jerky movements on the left side of his body and numbness followed by secondarily generalized tonic-clonic seizure (GTCs)and loss of consciousness lasting for a few minutes. According to the patient, these attacks started one year ago, with the last episode being two months prior. He also had a mild nonspecific headache with right frontal swelling for the last two months without any fever, cough, weight loss, and night sweats. There was no history of focal weakness, vision problems, or balance problems. He did not give a history of any myalgias, joint pain, dry eyes or mouth, skin rash, or oral ulcers. A review of the system was negative for any respiratory, gastrointestinal, and urinary symptoms. He did not take any medical treatment for these events. He was a nonsmoker, nonalcoholic, and denied any use of illicit recreational drugs. He was unmarried, and his family history was negative for epilepsy or any other neurological disease. He works as a cobbler in a leather factory. There is no sick contact history.

He was vitally stable. His neurological and systemic exam was unremarkable. He was started on phenytoin as an anti-seizure agent. Initial blood work including complete blood count, coagulation profile, blood chemistry, and renal and liver function tests did not show any significant abnormality. His chest x-ray showed hazy ill-defined shadows present in the right upper zone and to a lesser extent at the left upper zone and the right base. CT scan head non-contrast showed right side extra-axial hyperdensity along frontoparietal convexity of 12 to $13 \mathrm{~mm}$ in maximum thickness with effacement of the adjacent sulci and $5 \mathrm{~mm}$ midline shift to the left (fig1-a, b ) and was reported as Subdural hematoma. However, there was no history of any trauma or bleeding disorder. An osteolytic lesion in the right frontal bone (fig1-b, c ). There was no history of trauma and no clinical signs or laboratory abnormality indicative of bleeding diathesis for spontaneous subdural bleeding. MRI of the head with contrast was done after admission and the results showed: a right side intracranial extra-axial hemispheric crescentic shaped mass lesion showing low signal in T2, FLAIR, and T1 sequences with intense postcontrast enhancement and underlying dural involvement having a maximum thickness of $13 \mathrm{~mm}$, extending along the surface of the right cerebral hemisphere more at the higher cuts, reaching the ventral part of the interhemispheric fissure with mild left frontal dural enhancement as well. There was the associated irregularity of the adjacent inner skull table with right frontal focal full-thickness bony defect, underlying diploic and extracranial enhancing swelling as well as right frontal bone marrow signal abnormality bright in T2. Partial effacement of the right cerebral frontoparietal cortical sulci with mild compression of the right lateral ventricle and midline shift to the left side of about $5 \mathrm{~mm}$ was seen (fig2-a-e ).

The working differential diagnosis at the stage based on the clinical and imaging findings was an infective process (TB, fungal infections), neoplastic lesion (carcinomatosis, lymphoma, leukemia), histiocytosis, and inflammatory granulomatous processes like Sarcoidosis and Wegner granulomatous. Further work-up was tailored accordingly based on the differential. CT scan of chest, abdomen, and pelvis showed multiple enhancing pulmonary nodules of both lungs, in the upper lung lobe with no other abnormality in abdomen and pelvis (fig3-a, c ). Erythrocyte sedimentation rate was high 67 (reference 5 to 28), C - reactive protein (CRP)

129 (reference 1to 5), procalcitonin was normal whilst TB PCR was negative. The serum and urine protein electrophoresis were unremarkable. The flow cytometry indicated no definitive immunophenotypic evidence of a monotypic B-cell population. ANA and ANCA were negative.

He underwent right side craniotomy and had a right frontal skull osteolytic lesion with dural thickening. Resection of the thickened dural area and repair of skull defect were done. A Frozen Section Diagnosis of the skull lesion with intracranial subdural collection showed aggregates of chronic inflammatory cells, plasma cells, and lymphocytes, while that of thickened dura had dense fibrous tissue with a focal aggregate of chronic inflammatory cells. Histopathology Sections show sclerosed fibrous tissue in storiform pattern and patchy chronic inflammatory cell infiltrate, including lymphocytes, plasma cells, and few histiocytes and eosinophils. Few neutrophils were also noted. The inflammatory cells predominantly aggregated around blood vessels with no granuloma, necrosis, or atypia. Ancillary Immunohistochemistry studies with appropriate controls 
show the following results: CD138: Positive and highlighting an enormous number of plasma cells. IgG: Positive in plasma cells. IgG4: Positive in plasma cells (more than 10 positive cells/HPF). Kappa and Lambda: Polyclonal. Alk-1: Negative. S-100: Negative. Based on these findings definite diagnosis was an Inflammatory sclerosing pseudo tumoral lesion compatible with IgG4-related meningeal disease. Histopathology from CT-guided right lung biopsy was also consistent with Ig4 related disease.

Serum immunoglobulin measurement was positive for elevation in total IgE with a level $263 \mathrm{k}$ units/L (reference range between 0 and 114) and in IgG4 $(223 \mathrm{mg} / \mathrm{dL}$ with a reference range of 3-201). C3 and C4 194 and 193 were also elevated. Final cultures were negative for mycobacterium, fungal, and any other bacterial organism.

During his hospital stay, our patient had experienced episodes of sudden left side jerky movement with facial twitches followed by loss of consciousness. Levetiracetam was added. After the diagnosis of IG4 related disease, He was treated on oral steroids $60 \mathrm{mg}$ OD dose and IV $1 \mathrm{~g}$ rituximab dose was given to be followed by $2^{\text {nd }}$ dose in 14 days in his home country. On discharge, azathioprine $100 \mathrm{mg}$ OD was also prescribed He did not have further GTCs but reported episodic mild numbness of the left side with minor jerky movements. Inflammatory markers show CRP decrease to 53 . He was repatriated to his home country as he was a transit passenger.

\section{Discussion}

Meningeal involvement of IgG4-RD is rare and was first described in $2009(7,8)$. It is estimated to be slightly above $2 \%$ of overall clinical manifestations, based on prior studies (9). Fewer than 80 patients Ig4 related to HP have been described in the literature (1). It can mimic a variety of inflammatory, infective, neoplastic, and hemorrhagic conditions. IgG4RD-HP related diagnoses can be a diagnostic dilemma, despite a thorough clinical, laboratory, and imaging investigation.

Tissue biopsy remains the gold standard for the final diagnosis. Early clinical and etiological diagnosis is relevant to decisions about therapy. IgG4-HP closely follows granulomatosis with polyangiitis (GPA) as a leading cause of inflammatory HP. Other diagnoses to consider are lymphoma, sarcoidosis, tuberculosis, rheumatoid arthritis, and Langerhans-cell histiocytosis. (9).

There have been two from IgG4-RD HP described in the literature $(9,11)$. Isolated IgG4-HP was characterized by the absence of extra meningeal organ involvement (including the pituitary gland). Mutually exclusive lesions of the brain or spinal cord defined as "single-organ pachymeningitis." When extra-meningeal involvement was reported, IgG4-HP was defined as systemic IgG4-RD. Non-specific IgG4-HP was retained when data was lacking for classification.

IgG4-RDs occur, reported in men aged between 50 and 60 years (10), although our patient is much younger than that. The finding of an elevated serum IgG4 concentration is supportive of IgG4-RD but is not a constant finding (12). It can also increase in cancerous, allergic, autoimmune disorders, and other conditions. It should not be used as diagnostic criteria and evidence for starting empiric treatment. IgG4 levels are closely related to disease activity and the extent of organ damage (13). It is therefore essential to ascertain extra-neurologic organ involvement in patients with IgG4-HP presenting with high serum IgG4 levels and elevated plasma inflammatory markers. In such circumstances, PETCT has been shown to be of diagnostic value (9).

CSF alone is not a reliable parameter for the diagnosis of IgG4-HP. It is, however, common to find lymphocytic meningitis, and a moderate increase in CSF proteins with intrathecal synthesis of IgG with oligoclonal bands and increase in intrathecal IgG4 levels could be a surrogate marker for neurologic IgG4-RD when tissue biopsies could not be performed (13).

Intracranial hypertrophic pachymeningitis rarely accompanies subdural hygroma or hematoma. Only three cases have been reported in the literature, of which two cases involved a diagnosis of IgG4- RDs (14.15.16). Our case presented as an SDH mimic which is also rarely reported with HP. $(1,17)$ Skull involvement concomitant with IG4RD HP has been reported in a few cases $(6,18)$. These skull lesions are described as hyperostosis or thickening, while our case is unique in that it has lytic skull lesions. 
Pulmonary involvement reportedly occurs in $12-50 \%$ of patients with IgG4-related disease and takes the form of various sizes of lung nodules, lung masses, patchy ground-glass opacities, infiltrates resembling consolidation, reticular opacities, thickened bronchovascular bundles, central airway stenosis and obstruction, bronchiectasis, pleural effusion, nodular pleural lesions, and interstitial lung disease (19). In this case, however, there is the commitment involvement of Meningeal disease with pulmonary nodules which has been uncommon. In one report, a 48-year-old man presenting with headache was found to have HP along with incidental bilateral pulmonary nodules and had systemic features of both GPA and IgG4-related disease as well as

seropositivity for both cytoplasmic antineutrophil cytoplasmic antibodies (c-ANCA) and IgG4 (20). He was treated with prednisone and rituximab with improvement in his symptoms.

Steroids remain the first-line treatment (4). Short-term response to surgery in single-organ IgG4-HP is good and, in some cases, maybe sufficient to achieve remission (9). Although nearly all patients with IgG4-RD respond to glucocorticoids, approximately $40 \%$ of those that fail to achieve complete remission relapse within one year (21). Patients with multiorgan disease or an extremely high serum IgG4 are likely to require an agent other than glucocorticoids alone. Rituximab is preferred over other glucocorticoid sparing medications. It has been shown in I4G studies frequently as an excellent treatment for IgG4-RD and IG-HP among other immunosuppressive drugs $(22,23)$. It is important to follow this disease to look for new organ involvement in addition to recurrence.

\section{Conclusion}

We describe a unique case of a 25-year-old male with seizures whose clinical presentation was imitating an array of diseases making it a diagnostic challenge. Histopathology from two sites was performed; this aided in confirming the diagnosis of an IG4 related disease. This case reinforces the fact that it is crucial to consider the diagnosis of IgG4-RD in those presenting with pachymeningitis. A tissue diagnosis is imperative in this circumstance so that the appropriate management can be commenced, and complications avoided.

\section{Abbreviations}

IgG4 RD - Immunoglobulin G4-related disease

HP Hypertrophic Pachymeningitis

MRI: Magnetic resonance imaging.

CSF: Cerebrospinal fluid

\section{Declarations:}

Ethical approval: - The case report was approved by the Medical Research Centre of Hamad Medical Corporation (ABHATH) on June 16, 2021.Protocol ID MRC-04-21-559

\section{Consent for publication}

The patient gave written consent for their personal or clinical details along. with any identifying images to be published in this study.

\section{Availability of data and materials}

Not applicable

\section{Competing interests}

No conflict of interest.

\section{Funding}

This research did not receive any specific grant from funding agencies in the public, commercial, or not-forprofit sectors. 


\section{Authors' contributions}

MFW: case description, acquisition of data, analysis, and interpretation. LA: acquisition of data, analysis, and interpretation. AK and MA: critical revision of the manuscript for important intellectual content. The author(s) read and approved the final manuscript.

\section{Acknowledgements}

The publication of this article was funded by the Qatar National Library.

\section{References:}

1. Woo, P., Ng, B., Wong, J., Ng, O., Chan, T., Kwok, N. F., \& Chan, K. Y. (2021). The protean manifestations of central nervous system IgG4-related hypertrophic pachymeningitis: a report of two cases. Chinese neurosurgical journal, 7(1), 13. https://doi.org/10.1186/s41016-021-00233-5

2. Brito-Zerón P, Kostov B, Bosch X, Acar-Denizli N, Ramos-Casals M, Stone JH. Therapeutic approach to IgG4-related disease: A systematic review. Medicine (Baltimore). 2016 Jun;95(26): e4002. doi: 10.1097/MD.0000000000004002. PMID: 27368010; PMCID: PMC4937924.

3. Baptista B, Casian A, Gunawardena H, D'Cruz D, Rice CM. Neurological Manifestations of IgG4-Related Disease. Curr Treat Options Neurol. 2017 Apr;19(4):14. doi: 10.1007/s11940-017-0450-9. PMID: 28374231; PMCID: PMC5378735.

4. Hegade VS, Sheridan MB, Huggett MT. Diagnosis and management of IgG4-related disease. Frontline Gastroenterol. 2019 Jul;10(3):275-283. doi: 10.1136/flgastro-2018-101001. Epub 2018 Oct 31. PMID: 31288262; PMCID: PMC6583577.

5. Lu LX, Della-Torre E, Stone JH, Clark SW. IgG4-related hypertrophic pachymeningitis: clinical features, diagnostic criteria, and treatment. JAMA Neurol. 2014 Jun;71(6):785-93. doi: 10.1001/jamaneurol.2014.243. PMID: 24733677.

6. Lin CK, Lai DM. IgG4-related intracranial hypertrophic pachymeningitis with skull hyperostosis: a case report. BMC Surg. 2013 Sep 21; 13:37. doi: 10.1186/1471-2482-13-37. PMID: 24053604; PMCID: PMC3856477.

7. Wallace ZS, Carruthers MN, Khosroshahi A, Carruthers R, Shinagare S, Stemmer-Rachamimov A, Deshpande V, Stone JH. IgG4-related disease and hypertrophic pachymeningitis. Medicine (Baltimore). 2013 Jul;92(4):206-216. doi: 10.1097/MD.0b013e31829cce35. PMID: 23793110; PMCID: PMC4553969.

8. Chan SK, Cheuk W, Chan KT, Chan JK. IgG4-related sclerosing pachymeningitis: a previously unrecognized form of central nervous system involvement in IgG4-related sclerosing disease. Am J Surg Pathol. 2009 Aug;33(8):1249-52. doi: 10.1097/PAS.0b013e3181abdfc2. PMID: 19561447.

9. Levraut M, Cohen M, Bresch S, Giordana C, Burel-Vandenbos F, Mondot L, Sedat J, Fontaine D, Bourg V, Martis N, Lebrun-Frenay C. Immunoglobulin G4-related hypertrophic pachymeningitis: A case-oriented review. Neurol Neuroimmunol Neuroinflamm. 2019 May 7;6(4): e568. doi: 10.1212/NXI.0000000000000568. PMID: 31355304; PMCID: PMC6624094.

10. Lindstrom KM, Cousar JB, Lopes MB. IgG4-related meningeal disease: clinico-pathological features and proposal for diagnostic criteria. Acta Neuropathol. 2010 Dec;120(6):765-76. doi: 10.1007/s00401-010-0746-2. Epub 2010 Sep 16. PMID: 20844883

11. Umehara H, Okazaki K, Masaki Y, et al. Comprehensive diagnostic criteria for IgG4- related disease (IgG4-RD), 2011. Mod Rheumatol 2012; 22:21-30

12. Wang L, Chu X, Ma Y, Zhang M, Wang X, Jin L, Tan Z, Li X, Li X. A Comparative Analysis of Serum IgG4 Levels in Patients with IgG4-Related Disease and Other Disorders. Am J Med Sci. 2017 Sep;354(3):252256. doi: 10.1016/j.amjms.2017.05.009. Epub 2017 May 24. PMID: 28918831. 
13. Della-Torre E, Galli L, Franciotta D, Bozzolo EP, Briani C, Furlan R, Roveri L, Sessa M, Passerini G, Sabbadini MG. Diagnostic value of IgG4 Indices in IgG4-related hypertrophic pachymeningitis. J Neuroimmunol. 2014 Jan 15;266(1-2):82-6. doi: 10.1016/j.jneuroim.2013.10.008. Epub 2013 Oct 22. PMID: 24289956.

14. Miyazaki H, Tabuse M, Ishiyama N, Kikuchi R, Ogihara T, Nanki K. A case of multifocal fibrosclerosis presenting with chronic subdural hematoma. Brain and Nerve. 2011; 63:795 -9. 8. He Z, Ding F, Rong J, Gan Y.

15. A case of idiopathic hypertrophic cranial pachymeningitis presenting as chronic subdural hematoma. J Zhejiang Univ (Med Sci). 2016; 45:540 -3.

16. Polycystic subdural hygroma associated with immunoglobulin G4-related intracranial hypertrophic pachymeningitis: a case report Kazumichi Ota*, Yoshihiko Nakazato, Risa Okuda, Ryu Yokoyama, Hitoshi Kawasaki, Naotoshi Tamura and Toshimasa Yamamoto

17.Park IS, Kim H, Chung EY, Cho KW. Idiopathic hypertrophic cranial pachymeningitis misdiagnosed as acute subtentorial hematoma. Journal of Korean Neurosurgical Society. 2010 Aug;48(2):181-184. DOI: 10.3340/jkns.2010.48.2.181.

18. Lee YS, Lee HW, Park KS, Park SH, Hwang JH (2014) Immunoglobulin g4-related hypertrophic pachymeningitis with skull involvement. Brain Tumor Res Treat 2(2):87-91.

19. Raj R. IgG4-related lung disease. Am J Respir Crit Care Med. 2013 Sep 1;188(5):527-9. doi: 10.1164/rccm.201306-1121ED. PMID: 23992587.

20.Wyrostek S, Chakrabarti S, Baldwin K, Avila JD. A Case of Hypertrophic Pachymeningitis Associated with Immunoglobulin-G4 and c-ANCA. Case Rep Neurol. 2019 Oct 28;11(3):290-294. doi: 10.1159/000502569. PMID: 31762760; PMCID: PMC6872998.

21. Wallace ZS, Mattoo H, Mahajan VS, et al. Predictors of disease relapse in IgG4-related disease following rituximab. Rheumatology (Oxford) 2016; 55:1000-1008.

22. Ebbo M, Grados A, Samson M, et al. Long-term efficacy and safety of rituximab in IgG4-related disease: data from a French nationwide study of thirty-three patients. PLoS One 2017;12: e0183844. 29. Carruthers MN, Topazian MD, Khosroshahi A, et al.

23.Rituximab for IgG4-related disease: a prospective, open-label trial. Ann Rheum Dis 2015; 74:1171-1177.

\section{Hosted file}

Images_IgG4RD.docx available at https://authorea.com/users/445486/articles/544983-the-greatimposter-a-case-report-of-igg4-rd-hypertrophic-pachymeningitis-with-skull-lytic-lesionand-pulmonary-nodules 